\title{
Per-flow signalling extension across DiffServ domains
}

\author{
Giuseppe Bianchi \\ University of Palermo, Italy, bianchi@elet.polimi.it \\ Valentina Capaccio \\ CRES Palermo; INFN-CNAF; Italy, valentina.capaccio@cnaf.infn.it, \\ Nicola Blefari-Melazzi \\ University of Perugia, Italy, blefari@diei.unipg.it
}

\begin{abstract}
This paper describes a framework devised to extend per-flow admission control operation across Differentiated Services domains. Although the specific case of interoperability with RSVP is under discussion, our proposal can be easily adapted to other hop-by-hop signalling protocols. In our framework, DiffServ border routers accomplish three tasks. First, during the set-up phase, flows are mapped onto PHB groups on the basis of their QoS and traffic specifications. Second, signalling packets are tunnelled into IP packets marked as "probes", where the "probe" marking is a DCSP value associated to the considered PHB Group. Third, when the flow set-up is complete, flow data packets are marked as "data". The probe/data marking is built upon the Assured Forwarding PHB specification of DiffServ. QoS provisioning is achieved by tuning, within the DiffServ core routers, the dropping rates of AFmarked packets. Our approach provides a scalable solution to determine the decision of accepting/rejecting a transmission request with specified QoS requirements into the network, provisioning a per-flow call blocking service that spans heterogeneous IP domains.
\end{abstract}

Key words: Admission Control, Differentiated Services, RSVP

This research is supported by the European Community and MIUR in the frame of the POLLENS project (ITEA, if00011a)

The original version of this chapter was revised: The copyright line was incorrect. This has been corrected. The Erratum to this chapter is available at DOI: 10.1007/978-0-387-35620-4_43 


\section{INTRODUCTION}

Today, the Internet architecture provides only a best-effort service. If it is suitable for traditional Internet applications, it results inadequate for applications such as IP telephony, multimedia conferencing, audio or video streaming which are very sensitive to delay caused by network congestion. In order to provide Quality of Service, Internet domains should be given tools to control the level of congestion within the domain routers, and to react accordingly, e.g. by blocking incoming calls when congestion occurs.

The Integrated Service framework [1] was introduced in the mid of the 90's to accomplish the task of providing QoS. IntServ relies on a per-flow approach. It assumes that an explicit set-up mechanism (typically, but not necessarily, the RSVP protocol [2]) is used to convey information to routers so that they can maintain a per-flow state and provide requested services.

For several reasons, among which the lack of scalability of RSVP softstate management in high speed links, the IntServ model did not encounter a widespread deployment in the Internet. A different approach to support QoS, the Differentiated Service architecture [3], was more recently introduced with the goal of providing a scalable QoS provisioning approach, especially in the network core. Rather than IntServ flow-oriented approach, DiffServ is a packet-oriented approach, which does not recognize individual flows, but is aimed at handling aggregated flows. Packets are classified into a small number of "classes" on the base of the DiffServ Code Point (DSCP) contained in the header of IP packet. At each router in the DiffServ network region, packets are subjected to a "Per-Hop Behaviour" (PHB), which is associated to the DSCP label.

A problem of the DiffServ framework is the lack of a signalling mechanism able to convey the status of core routers to the edge nodes in order to take admission control decisions. In DiffServ regions, admission control is applied in a relatively static way by applying policing parameters at network elements. The customers of the DiffServ network regions and the owner of the DiffServ network region are expected to negotiate a static contract (Service Level Specification - SLS) for the transmission capacity to be provided to the customer at each of a number of standard DiffServ service levels. If it is true that static forms of provisioning do protect the network to some degree, they can be quite ineffective to prevent overload in a given service class. Hence, dynamic provisioning schemes should be considered for the DiffServ framework, as well as the interoperability of different QoS solutions in different domains.

The focus of this paper is to present a mechanism that allows that a perflow signalling protocol (such as RSVP) effectively interoperates with a stateless DiffServ domain. This topic has been tackled in [4], which presents 
a reference framework for IntServ/DiffServ interoperability. Indeed, when the problem of dynamically provision a DiffServ network is considered, the only proposed example solution is to rely on internal custom protocols devised to interact with an agent ("oracle", Bandwidth Broker) that has knowledge of resource availability and network topology to take admission control decisions.

The approach proposed in this paper is very different, as it does not require to introduce supplementary control entities within the DiffServ domain, but bases its operation on pure data plane mechanisms. Our starting point is a proposal for an admission control function over a DiffServ domain, called GRIP [5], originally intended for a pure DiffServ network (i.e. all the routers from source to destination are DiffServ). This proposal uses run-time traffic measurements to locally determine, in each core router, whether a new flow may be accommodated or it must be blocked, and makes smart usage of the Assured Forwarding PHB specification [6] to convey congestion information to the network edge where the admission control decision is actually taken. Scope of this paper is to describe the border router procedures necessary to accomplish interoperability of signalling protocols running in the outer domains, with a GRIP-based solution confined within the DiffServ domain.

The rest of the paper is organized as follows. Section 2 discusses the goals of the paper, and provides (section 2.2) a brief review of GRIP, the foundation upon which our framework is built on. Section 3 presents the border router upgrades that allow extension of per-flow signalling (and related QoS provisioning) across the DiffServ domain. Scalability issues are discussed in section 4. Finally, conclusions are drawn in section 5.

\section{MOTIVATION AND BACKGROUNDS}

To correctly grasp the motivations of this paper, it is fundamental to understand our different goals with respect to [4]. This RFC presents a framework for Integrated Services operation over Differentiated Services domains, The goal of [4] is to deploy end-to-end, quantitative QoS by applying the IntServ service models (namely, Controlled Load and Guaranteed Delay) end-to-end across a network containing one or more DiffServ regions. Signalling interoperability, i.e. the sequence of events in obtaining end-to-end QoS using RSVP through a DiffServ domain, is therefore a pure technical mean, in the context of [4], to provide an end-toend Integrated Services support model across DiffServ domains.

Conversely, rather than deploying a uniform QoS model across the Internet, we are interested in developing tools that allow the Internet to be 
equipped with a pure "call blocking service", regardless of the QoS provisioning level within each administrative domain. We argue that our view is much more similar to the actual Internet situation, and, perhaps, much more close to the ISP market interests. In fact, today ISPs are de-facto providing different levels of QoS support. For example, delays encountered across free of charge ISPs may be considerably higher than in premium ISP domains, which (in change of a possibly expensive charging) take care in keeping their network links underloaded. In this view, the introduction of signalling mechanisms, devised to block incoming calls when the QoS encountered in an administrative domain degrades ${ }^{1}$, is a very valuable tool.

\subsection{Reference scenario}

The reference network scenario considered in this paper is presented in figure 1. It consists of two Access Network Domains, at the network edge, separated by a DiffServ domain placed in the network core. We assume that both sending and receiving hosts, as well as routers in the Access Network domain, use an hop-by-hop signalling protocol to reserve resources across the network upon flow setup. Conversely, we assume that, in the DiffServ domain, only the Border Routers (BR1,BR2) are aware of such protocol. In what follows we'll assume that such a signalling protocol is RSVP (indeed, our described approach applies, with minimal modification, to alternative signalling protocols, provided they operate on an hop-by-hop basis).

The RSVP signalling extension proposed in this paper, is aimed at providing a per-flow call blocking service within the DiffServ domain, without violating the DiffServ principles. Our signalling extension proposal is sketched as follows (details in section 3). Upon arrival of an RSVP flow setup request at the DiffServ border router BR1 (see figure 1), the border router triggers a DiffServ-specific per-flow admission control function based on pure data-plane operation, presented in the following section 2.2. Unlike the traditional approaches to dynamic provisioning in DiffServ, such an admission control function does not requires interaction with an "oracle" (Bandwidth Broker), but bases the admission decision on the successful forwarding of suitably labelled packets through the DiffServ domain.

1 According to what said, QoS degradation is an ISP-specific concept: for a premium ISP, a link utilization above $50 \%$ may be considered a severe degradation, while for a free-ofcharge ISP even $80-90 \%$ utilization can be considered a good operational condition. 


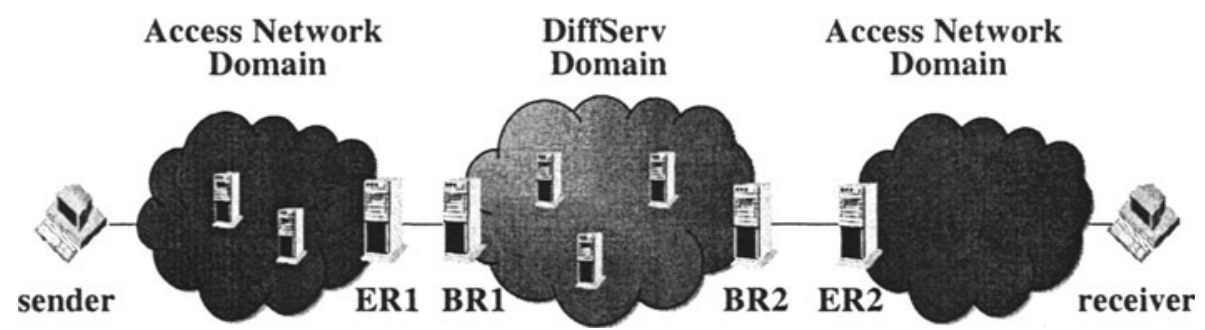

Figure1. Reference Network Scenario

The RSVP PATH message is thus encapsulated (tunnelled) in an IP packet; the packet is marked with a special DSCP, and delivered through the DiffServ region. If it is received at border router BR2, it is decapsulated and forwarded in the subsequent RSVP-capable network domain. A similar operation is performed on the returning RSVP-RESV message.

\subsection{Per-flow QoS support in the DiffServ domain}

Per-flow admission control in a stateless IP domain (e.g. DiffServ) has been object of thorough investigation $[7,8,9,10,11,12,13]$. These approaches range from mechanisms based on a pure endpoint operation down to lightweight signalling mechanisms which require some form of cooperation by the router, typically the execution of a measurement-based admission control (MBAC) mechanism $[14,15]$. An approach which allows the deployment of MBAC schemes over a fully conforming DiffServ network has been presented in [5] under the name GRIP (Gauge\&Gate Reservation with Independent Probing). As DiffServ routers support only data forwarding mechanisms, the idea is to a) implicitly convey signalling information via loss of packets, and b) drive such packet losses on the basis of run-time measurements locally taken by each network router. Such an approach can be supported over a standard DiffServ router by making smart usage of the Assured Forwarding (AF) PHB specification [6], as briefly reviewed in what follows (refer to [5] for details).

For convenience of presentation, assume two hosts connected via a single DiffServ domain. When the sender host wants to setup a one-way (e.g. UDP) flow with a receiver host, it sends a packet labelled as "PROBE" through the network. In the same time, a probing phase timeout is started. Each router along the encountered path applies the following trivial high-level rule:

- if (congestion at router greater than given level) then (drop incoming PROBE packets);

- else (let all PROBE packets cross through). 
It follows that if a PROBE packet is received at the destination, all the routers along the path are in uncongested situation, and the call can be accepted. This information is notified back to the sender via a FEEDBACK packet (or a set of packets, for improved reliability) transmitted at highest priority. Conversely, if at least one router is congested, no PROBE packet will be received at the destination; the probing phase timeout will elapse, and the call setup attempt will be aborted (alternatively, the source may reiterate the setup attempt according to some suitable backoff mechanism).

The (extremely simple) described operation leads to several design advantages. First, no requirement is imposed on the content of the PROBE and FEEDBACK packets, but the ability at each router to distinguish them and apply them a different forwarding behaviour (which is a native ability in DiffServ). Second, the notion of congestion which triggers the PROBE discarding mechanism at each router is left to each router (domain) administrator. As an example, a congestion detection mechanism might be implemented as an MBAC algorithm, i.e. based on run-time measurements taken on accepted data traffic. The congestion threshold is freely set by each domain administrator depending on its provisioning target.

Third, and most important, the described operation can be implemented via a smart usage of the AF PHB specifications. It simply suffices to reserve one or more AF classes (say class $\mathrm{x}$ ) for admission controlled traffic, and associate the following semantics to the AFxj DSCP labels: AFx1 for data packets and FEEDBACK packets; AFx2 for PROBE packets. The third available level, AFx3, is not strictly necessary and can be used for additional purposes [5]. The logical architecture of a router output port supporting AF is given in figure 2. Packets are dispatched to the relevant handlers based on their DSCP. The AFx class handler takes filtered measures ${ }^{2}$ on the AFx1 traffic (data packets), and, according to the outcome of such measures, it drives a dropping rule on the AFx2 traffic (PROBE packets). In the figure, we have used the term "gate" to visualize the dropping module, as this term highlights our usage of this module for blocking admission control requests.

2 Although, traditionally, AF PHB is considered synonymous of RED/RIO buffer management, this is a very restrictive interpretation of AF. Quoting [6], "an $A F$ implementation MUST detect and respond to long-term congestion within each class by dropping packets, while handling short term congestion (packet bursts) by queueing packets. This implies the presence of a smoothing or filtering function that monitors the instantaneous congestion level and computes a smoothed congestion level. The dropping algorithm uses this smoothed congestion level to determine when packets should be discarded". Indeed, we have evaluated the performance of GRIP over a RED implementation in [16], and we have found that, with tight RED threshold settings, even such an implementation is capable of providing reasonably good performance in light overload conditions (although severe performance impairments occur in overload). 


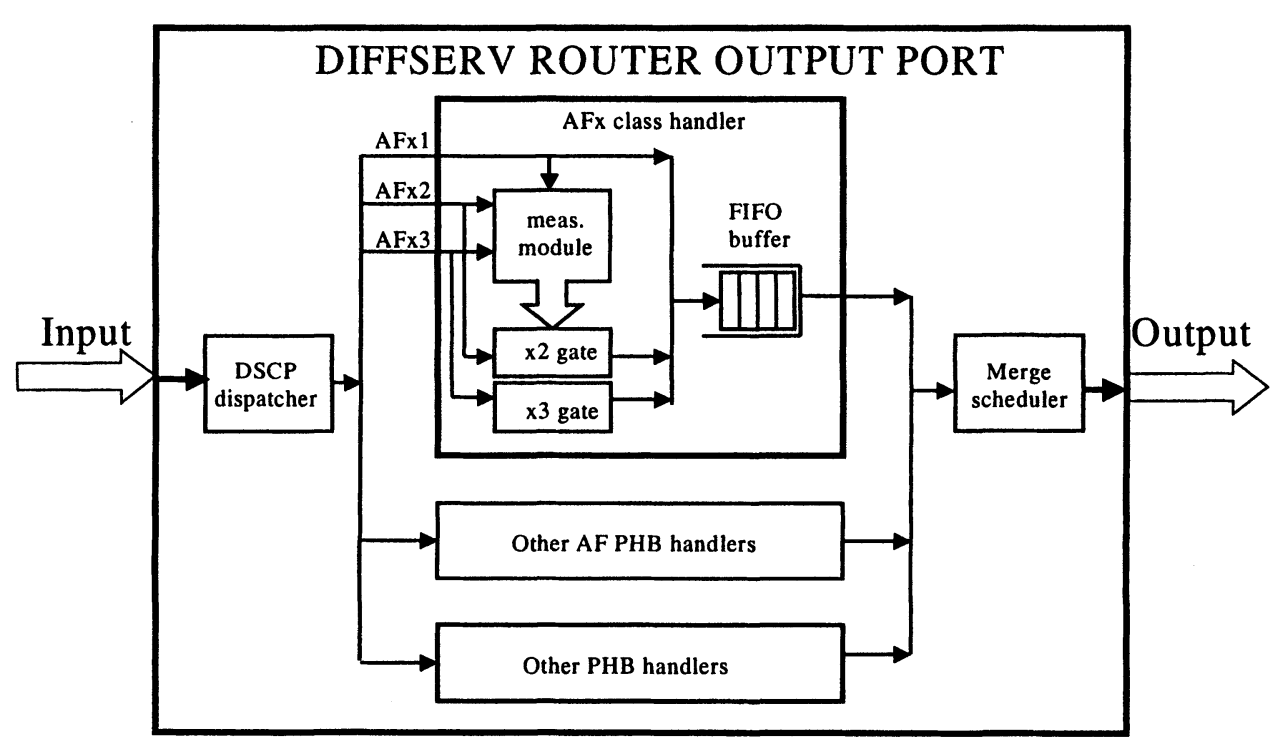

Figure 2. DiffServ router output port operation

\section{SIGNALLING EXTENSION ACROSS DIFFSERV}

With reference to the network scenario depicted in figure 1, The RSVP to DiffServ per-flow signalling conversion is managed by the Border Routers (BR1 and BR2), under the administrative control of the DiffServ domain. These BRs handle both RSVP and DiffServ signalling, and are responsible of the interoperation procedures described below.

\section{Step 1: flow to PHB group mapping}

BR1 operation starts as soon as an RSVP PATH message arrives from the sender. When RSVP carries a IntServ service requests, the RSVP packet payload specifies the sender traffic specification (TSPEC). Based on this field, and on additional reservation information carried by the PATH, the router selects the PHB group better suited to accommodate the flow, when/if accepted. The semantics of such a logical mapping are not specific part of the proposed signalling interoperation function, and are based on either endto-end QoS arguments, as well as internal DiffServ domain administrative management issues. For sake of an example, one of a possible mapping may be implemented as follows: 


\begin{tabular}{|c|c|c|}
\hline Flow-id & PHB group & status \\
\hline$\ldots \ldots$ & $\ldots \ldots$ & $\ldots \ldots$ \\
\hline SRC-IP SRC-port & $\begin{array}{l}\text { Data: } \\
\text { Probe: DSCP x1 }\end{array}$ & PATH_SENT \\
DST -IP DST-port & RESV_RECEIVED \\
\hline$\ldots \ldots$ & $\ldots \ldots$ & $\ldots \ldots$ \\
\hline
\end{tabular}

Figure 3. Logical Flow-PHB_Group mapping

- Based on additional QoS information (e.g. carried in the ADSPEC field ${ }^{3}$ ), BR1 determines whether there is at least one appropriately configured PHB Group within the DiffServ domain able to satisfy the sender requirements (if this is not the case, the call is blocked). Note that, in this phase, additional authorization and accounting information can be additionally taken into account.

- Based on the TSPEC, the BR1 selects the PHB group, among the available classes ${ }^{4}$, with the goal of supporting, within a PHB Group, flows with TSPECs as much similar as possible (e.g. maps incoming flows with peak rate in a given range onto the same PHB Group, to avoid that flows with widely different peak rates share the same group). This operation makes it easier to configure, for a given AF-PHB group, the congestion threshold (see section 2.2) within each core router.

A nice property of the described logical mapping stays in the fact that it is not RSVP specific, but it can be easily adapted to different QoS solutions (e.g. different ways of specifying traffic profiles and requirements) that will (might) be considered in the future activity about signalling (e.g. within the IETF NSIS group). It is simply necessary to update the mapping rules stored in a suitable BR information base.

The result of such a mapping is ultimately stored into a mapping table (figure 3) which associates the flow id (sender/destination IP/port pairs) to the selected PHB group (specifically, the set of DSCP values used by the

3 Ideally, this additional information is the RSVP RSPEC field. Unfortunately, in RSVP, this information is available only in the RESV message (i.e. RSVP receiver-oriented operation is in contrast with the natural sender-to-receiver PROBE direction). Hence, unless a second probing phase is started when the node receives the RESV message and the relevant RSPEC field, a flow to PHB group mapping precise enough to support the IntServ Guaranteed Service cannot be provided.

4 An important node is that, despite only four AF classes have been specified in [6], the DSCP assignment has a local scope within the administrative domain, i.e. an administrative domain has the freedom of defining supplementary PHBs / PHB groups inside the domain, as well as override the standardized DSCP values. 
DiffServ domain to support the PHB Group). An additional entry in the table is the status of the reservation process, which is initially set to PATH_SENT, meaning that the reservation process has been just started, but no information about the esit of such a process has been received yet. Regarding to implementation, the best place to store this table is in the policing module of the DiffServ border router. Moreover, a policy rule should be associated to the mapping table; specifically, if data packets arrive at BR1 while the status is still PATH_SENT, these packets should be dropped (or delivered as best effort, since flow setup has not been completed yet in the DiffServ domain.

\section{Step 2: BR1 signalling extension procedure}

Once a PHB group, say AF class $x$, has been selected, the RSVP packet is tunnelled into an IP packet. Such a packet is marked as PROBE (AFx2) and forwarded into the DiffServ network region. Tunnelling associated to AFx2 marking has the effect to extend the flow setup procedure also to routers within the DiffServ domain, as long as they support the AF behaviour described in section 2.2 .

As soon as the PROBE is sent, the BR starts a probing phase timer. This phase must be long enough to wait for the receipt of the RSVP RESV message that will be sent by the receiver host. If this message doesn't arrive at BR1 within the probing phase timeout, the BR assumes that the PROBE has been dropped because of congestion, and thus that the flow setup procedure needs to be aborted. Hence, it removes the relevant entry from the mapping table, and generates an RSVP PATHTEAR message that deletes the soft states installed in the routers of the upstream domain.

\section{Step 3: BR2 operation}

When the probe packet arrives at the border router BR2, it will extract the RSVP PATH message from the IP packet, process it according to standard RSVP processing rules and send it toward the receiver host. Unlike BR1, the BR2 does not need to store any additional state/table other than the standard RSVP state. In fact, when the PATH message arrives to the receiver host, it generates an RSVP RESV packet that follows the same path of the RSVP PATH packet, but in the reverse direction. As a RESV packet arrives at BR2, it is encapsulated into an IP packet, marked as highest priority ${ }^{5}$, and

5 This management for the FEEDBACK packet slightly differs from what stated in section 2.2. The reason is that, since BR2 does not store any mapping state, the information of which AF class were used to deliver the PROBE is lost. However, the only important issue regarding the FEEDBACK packet transmission, is reliability. Hence, the DiffServ administrative domain may associate a default mapping for all RSVP RESV packets onto the most reliable PHB available within the domain. 
sent to BR1 (since BR1 is an RSVP router, it is part of the path followed by the RESV message ${ }^{6}$ ). The encapsulated RSVP RESV message will traverse the DiffServ network region transparently.

Being BR2 an RSVP node, the RSVP protocol specifications still apply. In particular, if BR2 receives an RSVP RESV message, but doesn't contain a PATH state, it must generate a RSVP RESVTEAR message to delete specific reservation states. The only node that handles RESVTEAR messages in a special way is BR1 which, in addition to the standard RSVP operation, needs to delete the relevant entry from the mapping table.

\section{Step 4: BR1 operation with RESV messages}

When the border router BR1 receives a first RSVP RESV message (tunnelled in an IP FEEDBACK packet), it extracts the RSVP RESV message, resets the probing phase timeout, and updates status of the mapping table as RESV_RECEIVED. This status code carries the information that the DiffServ domain (as well as the subsequent domains) is able to accommodate the incoming flow. The RESV message is then processed by the RSVP part of BR1, and delivered to the sender, which starts transmitting data packets. As data packets arrive at router BR1, they are marked according to the DSCP value reserved for data packets (i.e. AFx1) stored in the relevant mapping table.

\section{SCALABILITY}

From the above description, it might appear that each DiffServ Border Router needs to support RSVP, or, in any case, a per-flow signalling approach that requires to store the flow to PHB Group mapping table given in figure 3. This would lead to severe scalability problems at border routers placed between two DiffServ domains (router R2 in figure 4), when interconnected at Gigabit speed. However, it is easy to understand that Border Routers between DiffServ domains do not need to manage flow states at all. The only requirement is to support a DSCP-to-DSCP mapping which translates the PHB Group used in one domain to carry admissioncontrolled traffic into the best fitting PHB Group used in the other. In essence, the described per-flow call blocking service is made able to cross the boundary between two DiffServ domain whenever they negotiate a static contract (i.e. a Service Level Specification) which matches the DSCP PROBE labels used in the different domains.

6 Note that it is not guaranteed that, within the DiffServ domain, the RESV message will follow the same path. Indeed, this is irrelevant to our solution, as core routers do not rely on reservation confirms - see section 2.2 . 


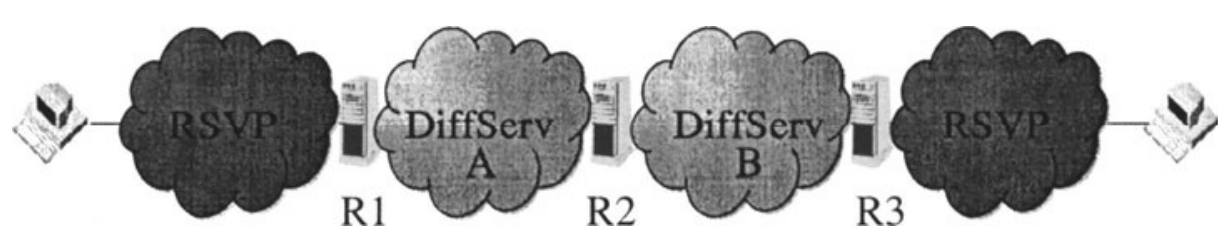

Figure 4. DiffServ to DiffServ interoperation

The operation described in the previous section is thus extended as follows. Consider an AFx2 (PROBE) packet travelling through the DiffServ domain A. This packet tunnels an RSVP PATH message, whose flow specification was mapped over AF class $\mathrm{x}$. When the packet arrives at router R2 (figure 4), it is simply remarked with a different DSCP to use in the subsequent domain $\mathrm{B}$. The unique requirement for the extension of the callblocking service is the condition that the new DSCP (say AFy2, from AF class y) must represents a PROBE also in the domain B. Note that the RSVP tunnel will be terminated only at router $\mathrm{R} 3$, which will perform the operation described for router BR2 in the previous section. Instead, the operation described for BR1 will be performed by router R1 only.

Finally, we remark that while the flow to PHB Group mapping at router $\mathrm{R} 1$ is performed on a per-flow basis, the PHB Group to PHB Group mapping at router R2 is statically specified. Hence, we achieve a per-flow admission control function where all the complexity (per-flow operation, and need to keep track of flow states) is pushed to the edge of the network.

\section{CONCLUSIONS}

In this paper, we have defined a framework to extend per-flow signalling across a DiffServ domain, to implement a scalable call-blocking service. Our approach requires the DiffServ border routers to accomplish the following tasks: 1) assign a Per-Hop-Behaviour Group to an incoming request, based on its QoS specification and on the QoS provisioned within the DiffServ domain; 2) tunnel signalling packets, suitably marked, through the DiffServ domain.

Our solution does not require the presence of centralized entities (such as Bandwidth Brokers) in the DiffServ domain. Being based on pure data forwarding operation (versus control-plane operation), our solution does not violate the DiffServ paradigm.

To provide a more detailed presentation, the investigation carried out in this paper has specifically considered the interoperation between RSVP and DiffServ. However, the key ideas of our proposal can be easily applied to 
any other hop-by-hop signalling protocol which can be considered in the future IETF activity about signalling (e.g. within the NSIS Working Group).

\section{REFERENCES}

[1] Braden, R., Clark, D., Shenker, S. : "Integrated Services in the Internet Architecture: an Overview”, IETF RFC1633, 1994.

[2] Braden, R., Zhang, L., Berson, S., Herzog, S., Jamin, S., "Resource Reservation Protocol (RSVP) Version 1 Functional Specification", IETF RFC2205, 1997.

[3] S. Blake, D. Black, M. Carlson, E. Davies, Z. Wang, W. Weiss: "An Architecture for Differentiated Services", RFC2475, December 1998.

[4] Bernet, Y., Yavatkar, R., Ford, P., Baker, F., Zhang, L., Speer, M., Braden, R., Davie, B., Wroclawski, J., And, E., Felstaine, “A Framework for Integrated Services Operation Over DiffServ Networks”, IETF RFC2998, November 2000.

[5] G. Bianchi, N. Blefari-Melazzi, "Admission control over assured forwarding PHBs: a way to provide service accuracy in a DiffServ framework", IEEE GLOBECOM 2001, San Antonio, Texas, November 2001, pp. 2561-2565

[6] Heinanen, J., Baker, F., Weiss, W., Wroclawski, J., “Assured Forwarding PHB Group”, IETF RFC2597, 1999.

[7] W. Almesberger, T. Ferrari, J.Y.Le Boudec: "SRP: a Scalable Resource Reservation Protocol for the Internet”, IWQoS'98, Napa (California), May 1998.

[8] I. Stoica, H. Zhang, "Providing Guaranteed Services without Per Flow Management", Proc. of ACM SIGCOMM 1999, Cambridge, MA, September 1999.

[9] L. Breslau, E.W. Knightly, S. Schenker, I. Stoica, H. Zhang: "Endpoint Admission Control: Architectural Issues and Performance", ACM SIGCOMM 2000, Stockholm, Sweden, August 2000.

[10] V. Elek, G. Karlsson: “Admission Control Based on End-to-End Measurements”, Proc. of IEEE Infocom 2000, Tel-Aviv, Israel, March 2000.

[11] G. Bianchi, A. Capone, C. Petrioli, "Packet Management Techniques for Measurement Based End-to-end Admission Control", KICS/IEEE Journal on Communications and Networking, June 2000.

[12] F.P. Kelly, P.B. Key, S. Zachary: "Distributed Admission Control", IEEE JSAC, Vol.18, No.12, December 2000.

[13] R. Mortier, I. Pratt, C. Clark, S. Crosby: "Implicit Admission Control", IEEE JSAC, Vol.18,No.12, December 2000.

[14] M. Grossglauser, D.N.C. Tse, "A Framework for Robust Measurement Based Admission Control”, IEEE/ACM Transactions on Networking, Vol.7, No.3, June 1999.

[15] L. Breslau, S. Jamin, S. Schenker: "Comments on the performance of measurementbased admission control algorithms", Proc. Of IEEE Infocom 2000, Tel-Aviv, March 2000.

[16] G.Bianchi, N. Blefari-Melazzi, V. Mancuso: "Endpoint Admission Control over Assured Forwarding PHBs and its performance over RED implementations", IWDC 2001, Taormina, September 2001, pp. 232-250. 